\title{
A Novel Approach for Lung Pattern Analysis using Neural Networks and Fuzzy Interface System
}

\author{
${ }^{1}$ N.Malligeswari, ${ }^{2}$ C.Rajani , ${ }^{3}$ Dr.G.Kavya \\ Department of Electronics and Communication Engineering \\ ${ }^{1,2}$ SKR Engineering College, ${ }^{3}$ SA Engineering College ,Chennai \\ malligeswari.n@gmail.com,rajaniece09@gmail.com
}

\begin{abstract}
An important and crucial aspect of image processing is effective identification of lung cancer at an initial stage. One of the state of the art methods in lung cancer detection is machine learning, namely ANNs (Artificial Neural Networks) and Fuzzy Logic. These researches mainly focus upon image quality and accuracy. ANN has proved to be efficient due to their ability to learn and generalize from data. To detect lung cancer based on fuzzy logic to classify the normal and abnormal images, in the abnormal result, use other symptoms as input to fuzzy logic system to find case of the patient (cancerous or noncancerous) depending on the

membership function of inputs. Expanding rough approximations into fuzzy environment which help to obtain solutions for various real time problems. Patterns are conferred to the network via the input layer which communicates to one or more hidden layers where the actual processing is done via a system of weighted connections. The hidden layers then bond to an output layer. The objective of the proposal is to materialize a means to fasten the process as well as the accuracy of detecting the cancer cells to a valuable extent it helps in saving human lives.
\end{abstract}

Keywords- Lung cancer, fuzzy system, Lung nodules, CT image, ANFIS, MDC.

\section{INTRODUCTION}

The most disastrous disease in human being is cancer among the people throughout the worldwide. Early diagnosis of interstitial diseases is very difficult for their treatment. Lung cancer is the main cause of cancer death and the contribution of lung cancer cited by world health organization is 1.3 million. This report also indicate that survival rate of cancer is only $14 \%$; but if it detected at an early stage the rate of survival can be increased up to $50 \%$. The main reason for high mortality rate due to lung cancer is it not easily detected in initial stage and very difficult to overcome at later stage. One Such field in automated diagnostic system design is Medical Image processing where numerous systems are proposed due explosive technology growth today.

In Lung cancer research one of the most sensitive methods for detecting cancer cell is Chest X-ray, Computed Tomography (CT) are the pulmonary standard for imaging. To analyses images at the lowest level Image processing is used to provide any quality. But the probabilities of image information do not increased by these operations but they decrease it if entropy is an information measure. Artificial Neural Network is a branch of Artificial intelligence and has been accepted as a new technology in computer science. Neural Networks are currently a 'hot' research area in medicine, particularly in the fields of radiology, urology, cardiology, oncology and etc. It has a huge application in many areas such as education, business, medical, engineering and manufacturing. Artificial neural networks are finding many uses in the medical diagnosis application. Neural Network plays an important role in a decision support system.

Objective of the project is to increase the visibility of the cancer nodule by proposing a new technique. Classifying lung tissue patterns using a deep Artificial neural network (ANN), with architecture designed for the specific problem. In this study, we put forward an enhanced method for training the proposed network by transferring knowledge from the related domain of general texture classification. Six publicly available texture databases are used to pretrain networks with the proposed architecture, which are then fine-tuned on the lung tissue data. Artificial Neural Network Fuzzy Interface System (ANFIS) has been innovatory step in precipitate detection of lung cancer. The proposed ANFIS system consist of five main steps : 1) Lung region extraction

2) Segmentation of extracted lung 3) lump detection 4) Feature extraction 5) Testing using Neural Network.

\section{EXISTING SYSTEM}

$\mathrm{N}$ Ganesanet al [1] made an attempt to make use of neural networks in the medical field (carcinogenesis (preclinical study)). In carcinogenesis, artificial neural networks have been successfully applied to the problems in both preclinical and post-clinical diagnosis. The foremost aim of research in medical diagnostics is to extend more costefficient and easy-to-use systems, procedures and methods for supporting clinicians. It has been used to analyze demographic data from lung cancer patients with a view to developing diagnostic algorithms that might improve triage practices in the emergency department. For the lung cancer diagnosis problem, the concise rules extracted from the network, achieve a high accuracy rate of on the training data set and on the test data set. 
Zhi-hua Zhou et al.[2002][2], stated that Lung Cancer is one of the most widespread and deadly disease in the world. Detection of lung cancer in its early stage is the key of its cure. In general, method for early stage lung cancer diagnosis mainly include those utilizing X-ray chest films, $\mathrm{CT}, \mathrm{MRI}$, isotope, bronchoscope, etc ., among which a very important measure is the so-called pathological diagnosis that analysis that analyzes the specimens of needle biopsies obtained from the bodies of the subjects to be diagnosed.

An automatic computer-aided detection (CAD) scheme for lung nodules recognition and $3 \mathrm{D}$ visualization is proposed using KNN classifier [3]. The nodule contours is extracted by active contour modelling and $3 \mathrm{D}$ visualization technique is applied on the segmented nodule to represent better visual results. Three dimensional segmentation methods for the identification of the pulmonary parenchyma in thorax X-ray computed tomography datasets was proposed

[4]. It is meant to be used as pre-processing step in the computer-assisted detection (CAD) system for malignant lung nodule detection that is being.

The automated system for nodule detection and classification is proposed [4] to read the CT images and to apply some advanced image processing principles to facilitate the segmentation and detection of mass lesions as well as small-sized cancer areas, presumably when they are biologically early in their evolution and amenable to surgical cure. The system is also able to display the size of the detected Cancer, to replace the manually process of measurements that is taken by the radiologist to measure the width and the length distances of the cancer area.

Suzuki Ket al [5] stated an image-processing technique for suppress the contrast of ribs and clavicles in chest radiographs by means of a multi resolution massive training artificial neural network (MTANN). An MTANN is a highly nonlinear filter that can be trained by use of input chest radiographs and the corresponding "teaching" images. "lung" images obtained by use of a dual-energy subtraction technique as the teaching images were employed. A validation test database consisting of 118 chest radiographs with pulmonary nodules and an independent test database consisting of 136 digitized screen-film chest radiographs with 136 solitary pulmonary nodules collected from 14 medical institutions are used in this study.

Hery Purnomo et al [6] explored the performance testing for PCNN method compared to the classical standard method for tuberculosis detection. There was significant improvement in processing time and Diagnosis percentage, which the image is processed first with Adaptive White Gaussian noise (AWGN) for reliability testing of the method.

Juan A et al [7] described some important aspects of recent visual cortex-based ANN models and finally discussed about the conclusions reached throughout the process.

$\mathrm{N}$ Ganesanet al [8] made an attempt to make use of neural networks in the medical field (carcinogenesis (pre-clinical study)). In carcinogenesis, artificial neural networks have been successfully applied to the problems in both pre-clinical and post-clinical diagnosis. The main aim of research in medical diagnostics is to develop more cost effective and easy-to-use systems, procedures and methods for supporting clinicians. It has been used to analyze demographic data from lung cancer patients with a view to developing diagnostic algorithms that might improve triage practices in the emergency department. For the lung cancer diagnosis problem, the concise rules extracted from the network, achieve a high accuracy rate of on the training data set and on the test data set.

\section{PROPOSED SYSTEM}

The first stage of the system is segmentation of lung region from CT images. Second part is to identify candidate nodules from segmented lung region section using rule based approach .Finally ANN is applied in order to classify the cancer. Initially extract the lung region from the CT image by using threshold and morphological reconstruction. After extracting the lung region, Segmentation is done to segment the lung part by using global threshold and morphological operation. The segmented nodules are used for feature extraction. The preprocessed image is then fed to feature extraction phase in which textual and structural features of nodule are extracted and fed to the classifier. The features like geometric and intensity-based statistical features are extracted.

The features extracted are given for classification. ANFIS based classifier is used to classify the cancerous and noncancerous nodules. It consists of Lung CT image, Global Thresholding \& Morphological operation, Feature extraction and ANFIS classifier models.

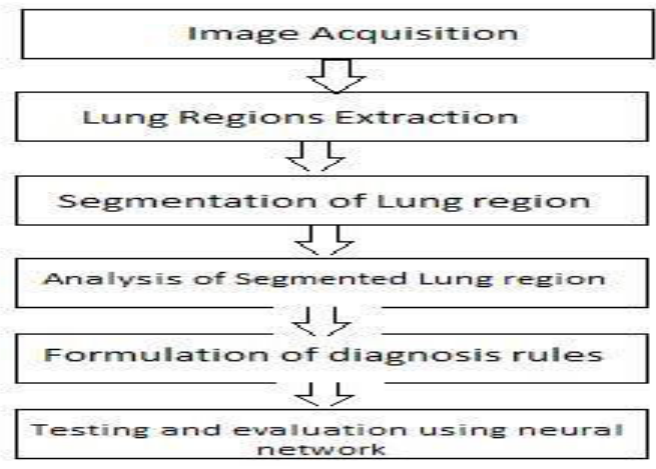

Figure 1. The Lung Cancer Detection System

\section{A. Lung Region Extraction}

When Compared to MRI images, lung CT images having low noise. So we can take the CT images for detecting the lungs. The main improvement of the computer tomography image having better Precision, low distortion and noise. The mean and Variance can be easily calculated. The calculated value is very closer to the original value.

\section{B. Lung CT Image Segmentation}

Segmentation of an image involves the separation of lung nodule from other part of the CT scan images and then enhancement of the resultant image to get details. This process includes series of steps which are listed below:

1) The input image is converted to gray image and Non Local Mean filter is applied to remove Gaussian white noise. 
2) Otsu's threshold is used to do segmentation of lung part from lung CT image.
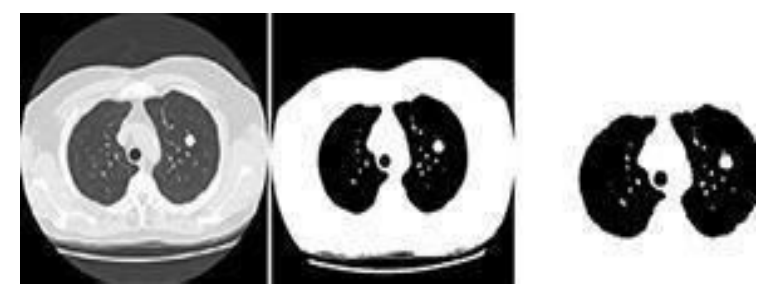

Figure 2. Segmentation: a) Original Image, b)Background removed Image, c)Threshold Image

\section{Feature Extraction}

The extracted features act as the root for classification process. These features are used to expand diagnostic rules to detect cancer nodule. The features that are used in this study are Area of candidate area Mean intensity value of candidate section Maximum drawable circle (MDC). The first feature is used to eliminate isolated pixels. The second feature helps in rejecting nodules which do not have chance to form candidate nodules.

\section{Formulation Of Diagnostic Rules}

After the required features are extracted the subsequent diagnostic rules are applied. The diagnostic rules help in reducing more number of false positive cancer nodules.

Rule 1: Initially the threshold values for area of candidate region are set $\mathrm{T} 1$ and $\mathrm{T} 2$. The threshold values are dogged by experiments. If the part of candidate region is less than or exceeds the threshold value then it is eliminated for further consideration.

Rule 2: The threshold value T3 is defined for MDC. If the radius of wanted circle is less than threshold value then candidate area is considered as normal cell and is eliminated for further consideration.

By implementing all the above rules the maximum regions which do not exhibit cancer nodule properties are eliminated. The results obtained by applying diagnostic rules are shown in the Fig 3. The Fig $3 \mathrm{~b}$ shows that the candidate regions reduce to two after the application of first rule.

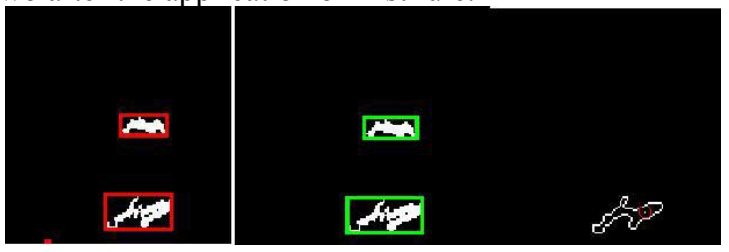

Figure 3. Application of diagnostic rule (3a) cancer nodule detected (3b) Application of first rule (3c)Application of second rule

\section{E. Classification Using ANN}

Artificial Neural Network is developed for identification and classification of candidate nodules obtained from application of diagnostic rules. ANN work by training and testing process applied to it. The ANN network consists of three main layers input layer, hidden layer, output layer. The network is trained using Back dissemination (BPD) algorithm. The idea of BPD is to reduce error produced by the variation between actual output and expected result [10].

Initially the finest optimized ANN is obtained by changeable various parameters of network like hidden nodes, guidance percentage for training ANN, number of epoch. After the thriving network has been developed it is then ready for classification process. The following Table 1 shows the result obtained from classification process of selected candidate nodule.

table I. Result of Classification Of Proposed Nodule

\begin{tabular}{|c|c|}
\hline Result & Nodule 1 \\
\hline Cancerous & $1(0.9892)$ \\
\hline Noncancerous & $0(0.7825)$ \\
\hline
\end{tabular}

\section{F. Anfis classifier models}

\section{1) Basic Global Thresholding:}

In one direction to decide a threshold is by visual scrutiny of the image histogram. The following iterative procedure is one such approach:

1. Select an initial ballpark figure for $\mathrm{T}$.

2. Portion the image using $T$. This will generate two groups of pixels: G1 consisting of all pixels with gray level values greater than $\mathrm{T}$ and $\mathrm{G} 2$ consisting of pixels with values less than $T$.

3. Calculate the common gray level values $\mu 1$ and $\mu 2$ for the pixels in the region G1 and G2.

4. Calculate a new threshold value:

$$
\mathrm{T}=\frac{1}{2}(\mu 1+\mu 2)
$$

5. Repeat steps 2 through 4 until the disparity in $\mathrm{T}$ in successive iterations is smaller than a predefined constraint T0. After thresholding morphological reconstruction operation is applied to extract the lung region alone.

\section{2) Morphological Reconstruction}

One of the functional applications of modernization is removing bits and pieces that pat the border of an image. Here, the key chore is to select the appropriate marker to realize the preferred effect.

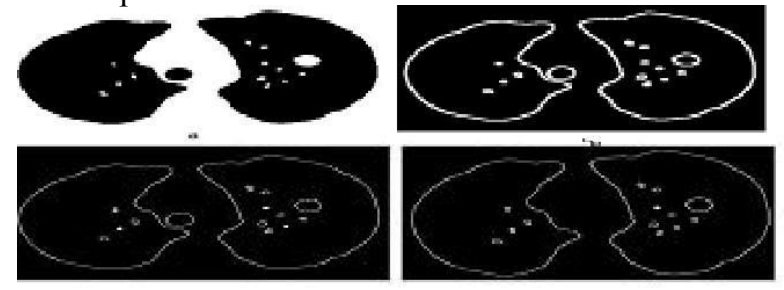

Figure 4. Post-processing Enhancement a)Morphological Operations, b) Border Detected Image c) Border Thinned Image d) Filled Image 
3) Lung Nodule Segmentation:

After extracting the lung region from the original image, by using thresholding operation the lung nodules are segmented. The threshold values obtained using global thresholding algorithm for lung nodule segmentation. After detecting the nodules, the nodule which is having less possibility to be cancer is filtered out using morphological opening

\section{EXPERIMENTAL RESULT}

In this research Mat lab software is used to program and test the system. The system doesn't require any radiologists to mention down the cancerous part of the image. After the execution of the program the geometric and the intensity features are obtained as output. While considering the arithmetical features if the area and perimeter is high the nodule is confirmed as cancerous. If the area is greater than $30 \mathrm{~mm}$, nodule is cancerous. While taking into account the intensity values contrast and mean should be high for cancerous nodules. Contrast might be greater than $0.05 \mathrm{~mm}$ and mean might be greater than $0.005 \mathrm{~mm}$. Otherwise the nodules are non-cancerous. The proposed method is evaluated by the Lung image database consortium [11] database, a publicly available database from the National Biomedical Imaging Archive (NBIA). In this database its nodules have been fully annotated by multiple radiologists. The LIDC database consists of $84 \mathrm{CT}$ scans, but only $58 \mathrm{CT}$ scans contain nodules. In the nodule containing CT scans, we randomly collected 15 CT scans in order to evaluate the proposed system. There were about 200 slices per scan. Testing of ANFIS for 60 Training data and 20 Training data is shown in figure 2 and figure 3 respectively. The classification result of sample 1 and sample 2 is given in the figure 4 .

\section{Simulation OUtPut}

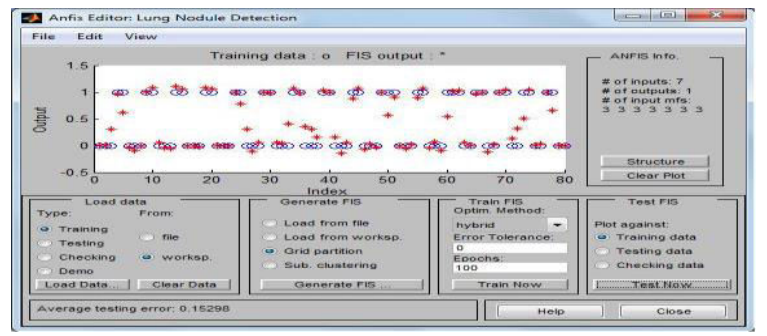

Figure 5. Testing ANFIS with 60 Training Data

The various Performance Metrics (Accuracy, Precision, Recall, and Specificity) for the test data are shown in Table

2. The tabulations are shown in percentage, each column indicates the Classifier used and the rows indicate the Metric value.From table 2 it is shown that accuracy of ANFIS is $96.23 \%$ which is better that ANN (92.68\%) and kNN (85.37\%). ANFIS predicts images of stage I and stage II more accurately.

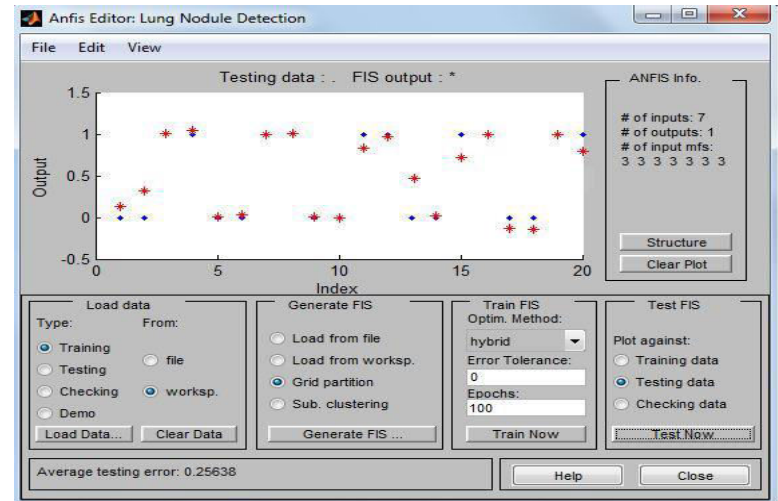

Figure 6. Testing ANFIS with 20 Testing
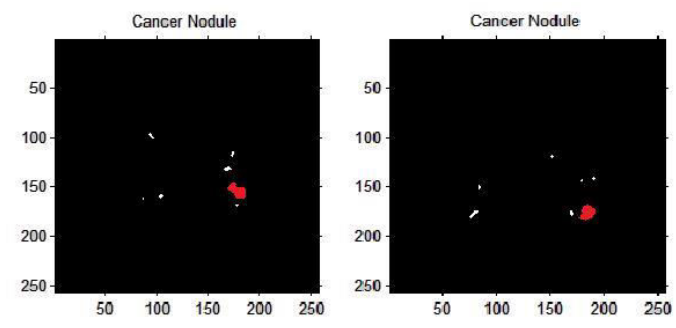

Figure 7. Classification Result (a) Sample 1 (b) Sample 2

TABLE II.

PERFORMANCE METRICS FOR TEST DATA

\begin{tabular}{|c|c|c|c|c|}
\hline \multicolumn{5}{|c|}{ Classifier } \\
\hline & & ANN & KNN & ANFIS \\
\hline \multirow{4}{*}{ Metrics } & Accuracy(\%) & 92.68 & 85.37 & 96.23 \\
\hline & Precision(\%) & 87.50 & 84.62 & 88.25 \\
\hline & Recall(\%) & 100.00 & 91.67 & 100.00 \\
\hline & $\begin{array}{c}\text { Specificity } \\
\text { (\%) }\end{array}$ & 100.00 & 76.47 & 100.00 \\
\hline
\end{tabular}

\section{CONCLUSION}

The field of Disease Diagnosis is an always developing and very active field of research. The purpose of the current study was to forecast the condition of patient for early detection of lung cancer. A novel approach for predicting Lung cancer nodules as cancerous and non-cancerous using ANFIS based Classifier. The approach starts by extracting lung area. The analytical rules were used to eradicate false positive candidate nodules stage using ANN and ANFIS Classifier. The best ANFIS architecture was developed and used for classification of lung cancer nodules in CT images. The system helps the physician and the radiologist to recognize the doubtful nodules and thus to increase the sensitivity of the diagnosis. An assessment of classification accuracy for ANN, KNN and ANFIS Classifiers was made on lung CT scan images of stage I and stage II. The 
classification rates obtained for the ANFIS, ANN and k-NN Classifier were $96.68 \%, 85.37 \%$ and $96.23 \%$ for the test images. The future extent of this project is to enlarge the number of features and from that the superlative features will be selected using some optimization techniques.

\section{REFERENCES}

[1] Dr. N. Ganesan, Dr.K. Venkatesh ,Dr. M. A. Rama ,"Application of Neural Networks in Diagnosing Cancer Disease Using Demographic Data", International Journal of Computer Applications, Volume. 1, No. 26, pp.76-85.

[2] JIA Tong, ZHAO Da-Zhe, WEI Ying, ZHU Xin-Hua, WANG $\mathrm{Xu}$, "Computer-Aided Lung Nodule Detection Based On CT Images", IEEE/ICME International Conference on Complex Medical Engineering, 2007.

[3] Soltaninejad, S, Keshani, M. ; Tajeripour, F. "Lung nodule detection by KNN classifier and active contour modelling and 3D visualization", Artificial Intelligence and Signal Processing (AISP), 6th CSI International Symposium, 2012, pp. 440 - 445

[4] C. Clifford Samuel, V. Saravanan, M. R. Vimala Devi, “ Lung Nodule Diagnosis from CT images using Fuzzy Logic", ICCIMA '07 Proceedings of the International Conference on Computational Intelligence and Multimedia Applications, 2007, vol.3, pp.159-163..

[5] Suzuki K, Abe H, MacMahon H, Doi K, "Image-processing technique for suppressing ribs in chest radiographs by means of massive training artificial neural network (MTANN)". IEEE Transactions on Medical Imaging., 2006, vol. 25,no.4, pp. 406416.

[6] Hery PURNOMO, Hiroshi, HASEGAWA, Kazuo, SHIGETA and Hideya TAKAHASHI, "Pulse Coupled Neural Network for Identifying the Tuberculosis on Human Lung" Volume 44, 2003, pp. 23-30.

[7] Juan A. Ramírez-Quintana, Mario I. Chacon-Murguia and Jose F. Chacon-Hinojos, "Artificial Neural Image Processing Applications: A Survey", Engineering Letters,2009, volume 20, issue 1 , pp 68-80.

[8] Dr. N. Ganesan, Dr.K. Venkatesh ,Dr. M. A. Rama,"Application of NeuralNetworks in Diagnosing Cancer Disease Using Demographic Data", InternationalJournal of Computer Applications, Volume. 1, No. 26, pp.76-85.

[9] Dilip Roy Chowdhury, MridulaChatterjee\& R. K. Samanta, “An Artificial NeuralNetwork Model for Neonatal Disease Diagnosis", International Journal of ArtificialIntelligence and Expert Systems (IJAE), Volume 2, Issue 3, 2011.

[10] Azian azamimi Abdulla, Syamimi Mardiah Shaharum," Lung Cancer Cell Classification Method using Artificial Neural Network", information Engineering Letters ,Vol. 2 No.1 March 2012.

[11] S.G.Armato, G.McLennan, M.F. McNitt-Gray, C.R.Meyer, D.Yankelevitz, D.R.Aberle, C.I.Henschke, E.A.Hoffman, E.A.Kazerooni, H.MacMahon, A.P.Reeves, B.Y.Croft, L.P.Clarke

(2004) L.I.D.C.R. Group, Lung image database consortium: developing a resource for the medical imaging research community, Radiology 232, 739-748. 